\title{
ON THE PARADOXICAL ASPECTS OF NEW QUANTUM EXPERIMENTS
}

\author{
L. Vaidman \\ School of Physics and Astronomy \\ Raymond and Beverly Sackler Faculty of Exact Sciences \\ Tel-Aviv University, Tel-Aviv 69978, Israel
}

\begin{abstract}
Two recently proposed quantum experiments are analyzed. The first allows to find an object without "touching" it. The second allows to teleport quantum states, transmitting a very small amount of information. It is shown that in the standard approach these experiments are in conflict with the intuitive notions of causality and locality. It is argued that the situation is less paradoxical in the framework of the many-worlds interpretation of quantum theory.
\end{abstract}

I shall discuss two recently suggested quantum experiments. These experiments lead to paradoxical situations. I will argue that in the framework of the Many-Worlds Interpretation of quantum theory the paradoxes do not arise.

The first experiment is the "interaction-free measurement" (Elitzur and Vaidman 1993). The experimental group at Innsbruck headed by Prof. Zeilinger is working now on its realization. Before describing the experiment let me discuss a general question: "How do we know that there is an object in a region of space?"

The simplest case is when an object itself causes some physical changes outside the region:

i) The object is charged, so there is a field outside. We can measure this field.

ii) The object yields a potential outside. Aharonov and Bohm have taught us that potential even without field leads to a measurable effect.

iii) The objects radiates photons or other particles.

If there is nothing, then, of course, none of (i)-(iii) will occur. However, even if there is something in the region, it might happen that there will be no field, no potential, and no radiation outside. The object may be neutral. The object must have mass, and therefore it must create a gravitational field. But let us assume that the field is too weak to detect. (Another way to eliminate signals from the gravitational field is to consider a compact sphere of mass $M$ or a large spherical shell of the same mass. Both produce the same gravitational field (and potential) outside the region.)

If (i)-(iii) did not occur, how can we know that the object is in the region? There is one simple case when getting information without "touching" is possible. Suppose we know that an object is located in one of two boxes. If we open one of them, it might be empty. Surely we have not touched the object; nevertheless, we know now that the object is in the second box. Quantum mechanics allows a subtle variation of this case. We can (in principle) prepare a two-particle system in a correlated state such as the Einstein-Podolsky-Rosen (EPR) state. Then, measuring the location of one particle yields the location of the other even though we have not touched it. In both cases we had prior information about the object. Here we consider the situation in which we do not have any prior information.

In order to explain the meaning of a measurement without "touching", assume that the object which we are trying to locate in a certain region is a supersensitive detector, one with $100 \%$ efficiency for all kinds of particles. Nowadays experimentalists can create a single-photon state. Every time such a photon reaches the detector, the detector clicks. Applying standard logic - if A implies B, then negation of B implies negation of A - we can claim that if the detector did not click, then no particles reached it.

Locating of such a detector without triggering it leads to a paradox: we obtain information about a region of space from which nothing came out. Indeed, we assumed that the detector itself sends nothing out, and since it did not click, no particle from the outside visited the region.

Let us now present Elitzur-Vaidman solution of this task. We call it an "interaction-free" measurement. I have to mention from the outset that the measurement is not always successful. At least in half (on average) of the cases we do touch the object, and if the object is a super-detector it will click in all these cases. Still we have finite chance for success: in up to $50 \%$ of the cases the detector does not click but we are $100 \%$ sure that something is inside the region.

Our method employs the Mach-Zehnder interferometer used in classical optics. In principle, it can work with any particle. The particle reaches the first beam splitter which has transmission coefficient $\frac{1}{2}$. The transmitted and reflected parts of the particle's wave are then reflected by the mirrors and finally reunite at another, similar beam splitter (Fig. 1a). Two detectors collect the particles after they pass through the second beam splitter. We can arrange the positions of the beam splitters and the mirrors such that, due to destructive interference, no particles are detected by one of the detectors, say $D_{2}$, and all are detected by $D_{1}$. We place the interferometer in such a way that one of the routes of the particle passes through the place where the super-detector might be (Fig. 1b). We send a single particle through the system. If the interferometer is empty, than detector $D_{1}$ clicks. But if the super-detector 
blocks one arm of the interferometer than there are three possible outcomes of this measurement:

i) super-detector clicks, ii) detector $D_{1}$ clicks, iii) detector $D_{2}$ clicks.

The probability for the first case is $\frac{1}{2}$. In the second case (for which the probability is $\frac{1}{4}$ ), the measurement does not succeed either. The particle could have reached $D_{1}$ in both cases: when the super-detector is, and when it is not there. Finally, in the third case, when the detector $D_{2}$ clicks (the probability for which is $\frac{1}{4}$ ), we have achieved our goal: we know that the super-detector is inside the interferometer and it did not click. A slight modification allows us to find the detector without triggering it with the probability arbitrarily close to 50\% (Elitzur and Vaidman 1993).

Note that we have succeeded to find out that a region of space is not empty without any particle passing through it. But we cannot find out that the region is empty without passing a particle through the region. Indeed, we know that the region is empty when, after passing very many photons through the interferometer, the detector $D_{2}$ remains silent. In this case we have no reason to claim that the photons have not passed through the observed region.

In this example we have presented the following paradox: nothing inside the region had any influence on the world outside, nevertheless, we obtain certain information about what is inside.

Let us describe now the second paradox. We will discuss a recent proposal of Bennett et al. (1993) for teleportation of an unknown quantum state. They found a method for transmitting an unknown quantum state of a spin- $1 / 2$ particle to a distant spin-1/2 particle without actually moving the particle from one place to another. Recently, I have found a way to generalize teleportation procedure to systems with continuous variables (Vaidman 1994).

The word "teleportation" recalls the heroes of Star Trek entering a transmitting cabin in their starship Enterprise: in a few seconds they disappear and immediately appear on a distant starship. It sounds, however, like a bad science fiction: too many laws are broken in this picture. For instance, the center of mass of a closed system should not move. But, when the heroes move far away to another starship, the center of mass of the closed system consisting of the two starships does move. However, quantum theory teaches us that the essential feature of an object is not the matter out which it is made, but its form. Indeed, all objects are "made" out of identical elementary particles, and what distinguishes one object from another is the state of these particles. Thus, we can consider a different kind of machine for teleportation. The receiving teleportation chamber is not empty before the transmission, but it contains elementary particles in a number equal to the number of particles of the object to be transmitted. Then, the transmission results in building the Star Trek heroes out of these particles, while the heroes in the cabin on Enterprise revert to an unstructured set of elementary particles.

One might be tempted to build the heroes in several locations, i.e., to produce several copies. This is certainly not a teleportation. But the unitarity of quantum theory prevents this possibility. It is impossible to clone an unknown quantum state. It is also impossible to identify an unknown quantum state without significantly changing it. Therefore, the only option quantum mechanics leaves, is destruction of the heroes in one place and their creation in another; i.e., teleportation of an unknown quantum state of about $10^{26}$ particles to an identical set of particles located in a distant region. Clearly, this is not a feasible project today, but the teleportation of a quantum state of one particle is a subject of serious consideration of many experimentalists.

In order to teleport a quantum state $\Psi$ from one place to another we need a "quantum channel". This is a system of two completely correlated particles, one located in a place from where we want to teleport the state and the other is in the remote location. The first step of the teleportation procedure is a particular local quantum measurement performed on the system of two particles: the particle in the state $\Psi$ and the first particle of the correlated pair. In the case of teleportation of a spin state of a spin-1/2 particle, this measurement has four possible outcomes; let us label them as numbers from 0 to 3. The next step of teleportation is a "very short" telephone call to the location of the second particle of the pair for transmitting the result of our measurement, a number from 0 to 3 . The last step is performing a "rotation" of the state of the second particle. There are four cases: If the transmitted number is zero, no rotation is required, the teleportation has been completed. In all other cases we have to rotate the state by an angle $\pi$ around $x, y$, or $z$ axes, in accordance with the outcomes 1,2 , or 3 . After the appropriate rotation the state of the second particle is $\Psi$.

The procedure for teleportation of a quantum state of a continuous variable $\Psi(q)$ is very similar. We use again the EPR pair of correlated particles as a "quantum channel". We perform a local measurement on a system which includes a particle in a state $\Psi(q)$ and one member of the EPR pair. After the local measurement, which yields two numbers $a$ and $b$, the state of the second particle is $e^{i b q} \Psi(q+a)$, i.e., the original state shifted in $q$ and in the conjugate momentum $p$. In the next step we transmit the numbers $a$ and $b$ by phone. The last step is a back shift of $a$ in $q$ and $b$ in $p$, thus bringing the second particle of the pair to the state $\Psi(q)$.

I see a paradoxical situation in this example as follows. Special theory of relativity tells us that nothing can move faster than light. Any massive body cannot move faster than light, and no information can be sent faster than light. However, the phase velocity of waves, for example, can move faster than light. As I see it, the spirit of the theory of relativity is that nothing which has "direct physical meaning by itself" should move faster than light. Conversely, only things which cannot move faster than light can have any physical meaning.

All objects are "made" out of identical particles, so the particles cannot characterize the object. The essence of an 
object, then, is in its quantum state. But in the process of teleportation of a quantum state the only part which cannot be sent faster than light is a small amount of classical information which we have to transmit by phone. Therefore, it seems that we have to admit that the essence of a quantum state is in this classical information. The spin-state of a spin-1/2 particle is described by a direction in a three-dimensional space, i.e., by two continuos variables, the two angles. Nevertheless, in teleporting this state we have to transmit only a number from 0 to 3 , just two bits of information! For the case of a state with continuous eigenvalue the description is a continuum of complex numbers, while we have to transmit only two numbers.

Let me repeat the logic of the argument. The theory of relativity teaches us that we cannot move anything essential faster than light. In the process of teleportation of a quantum state, say of a spin- $1 / 2$ particle, the limitation on the velocity of transmission is on just two bits of classical information. Therefore, the essence of the quantum state of a spin is only the two bits. This however, is in conflict with the fact that the description of the state requires two real numbers.

The first step of the teleportation is closely connected to a paradoxical situation which is so much discussed that there is a consensus of agreement not to agree about its interpretations. Consider EPR-Bohm pair of (anti)correlated spin$1 / 2$ particles. Each particle has no quantum state by itself, each one is in a "mixed" state. After the measurement of a spin component of particle 1, the EPR-Bohm state collapses into a product state. Immediately after the measurement each particle is in a pure state. One can consider the measurement of the spin of particle 1 as a creation of its spin state and immediate teleportation of the (flipped) state to the second particle (without destruction of the state of the first particle). In this case there is no need for sending any classical information. This kind of instantaneous teleportation clearly contradicts the spirit of the theory of relativity. Let me note that there is no contradiction with the letter of Einstein's theory: there is no way of sending information faster than light using collapse of the quantum wave function.

I want to argue that the paradoxes presented above are resolved, or at least appear less paradoxical in the framework of the Many-Worlds Interpretation (MWI) (Everett 1957). The MWI itself has several interpretations, some of which conceptually different. I (Vaidman 1993) take a view in which we have one physical universe which incorporates many (subjective) worlds. The physical universe is described by one wave function, which evolves deterministically according to the Schrödinger equation. This wave function can be decomposed into a superposition of many states, each corresponding to a different story. One of the stories is the world as you, the reader of this paper, know it. What we perceive is just a small part of what is in the universe. The laws of physics relate to the whole universe, and it is not surprising that consideration of only a part of it leads to paradoxical situations. Considering all worlds together, the physical universe, resolves the paradoxes.

Let us turn now to the example of the interaction-free measurement. In the framework of the MWI it is not true that we got information about the region without anything being there. The photon which we sent into the interferometer was there, but - in another world. In our experiment three worlds (three different stories) appear: (i) the super-detector clicks, (ii) detector $D_{1}$ clicks, and (iii) detector $D_{2}$ clicks. Obtaining information in the world (iii) without any object being in the region became possible because in the world (i) a photon was in the region (and it triggered the super-detector).

Now it is clear why we could not get information that the region is empty without a photon being there. In this case there is no another world, except the one we are aware of, so obtaining information about the region without being there is on the level of the whole universe. Our physical intuition correctly tells us that such situation is impossible.

Let us turn now to the second example. First, consider the paradox arising from the first step of teleportation. The paradox is that a measurement at one location instantaneously transmits a quantum state (sometimes transformed in a known way) to a place far away. The answer in the framework of the MWI is that nothing is transmitted by the local measurement. At the beginning the system in the remote location was in a mixed state corresponding to a certain density matrix. The local measurement in another location does not change this mixed state. The only change is that before the measurement the remote system was correlated only to a similar system, while after the measurement it is correlated also to a macroscopic measuring device and to an observer. Thus, the local measurement splits the world into a number of worlds. The superposition of these worlds yields the density matrix of the remote system which is the same as the original one. This is why we cannot send superluminal signals via the (apparent) collapse of a quantum state.

Let us turn now to the example of teleportation per se. The first step of teleportation of a state $\Psi$, the local measurement, splits the world according to the possible outcomes of the local measurement. After completing the teleportation, we obtain in the remote location the state $\Psi$ in all worlds. Therefore, in the whole universe (assuming that before the measurement was just one world in the universe), the state has been transmitted from one particle to another. In this case the MWI does not tell us that the paradoxical situation does not exist on the physical level of the universe. The teleportation works not only in a world, it works in the whole universe! The MWI provides another explanation. Let us discuss it now.

In the framework of the MWI the teleportation procedure does not move the quantum state: the state was, in some 
sense, in the remote location from the beginning. The correlated pair, which is the necessary item for teleportation, incorporates all possible quantum states of the remote particle, and, in particular, the state $\Psi$ which has to be teleported. The local measurement of the teleportation procedure splits the world into worlds in all of which the state of the remote particle differs form the state $\Psi$ by some known transforamtion. The number of such worlds is smaller than the necessary information for defining the state $\Psi$. This explains why the information which has to be transmitted for teleportation of a quantum state - the information which world is it (what tranformation has to be done) - is much smaller than the information which is needed for creation of such a state. For example, for the case of a spin-1/2 particle there are only 4 different worlds, so in order to teleport the state we have to transmit just 2 bits.

The MWI clarifies the issue of nonlocality in quantum mechanics. The quantum state of the universe, as well as its components which correspond to various worlds, are nonlocal. Physical interactions changes locally the state of the universe. If there are no splittings of worlds, the states corresponding to the worlds are changed locally too. Measurements are interactions which split the worlds into more worlds. These new worlds differ one from another not only in the region of the measurement, but also in other places. This happens due to the correlations incorporated in the quantum state before the measurement. Thus, in the created worlds we obtain, effectively, nonlocal changes, while there is no nonlocal action on the physical level of the universe.

It is a pleasure to thank Yakir Aharonov for stimulating discussions. The research was supported in part by grant 425/91-1 of the Basic Research Foundation (administered by the Israel Academy of Sciences and Humanities).

\section{References}

Bennett, C.H. Brassard, G. Crepeau, C. Jozsa, R. Peres, A. and Wootters, W.K. (1993), "Teleporting of Unknown Quantum State via Dual Classical and Einstein-Podolsky-Rosen Channels", Phys. Rev. Lett. 70, pp. 1895-1898.

Everett, H. (1957), "Relative State Formulation of Quantum Mechanics", Rev. Mod. Phys. 29, pp. 454-462.

Elitzur, A. and Vaidman, L. (1993), "Quantum Mechanical Interaction-Free Measurements", Found. Phys. 23, pp. 987-997. Vaidman, L. (1993), "On Schisophrenic Experiences of the Neutron or why We should Believe in the Many-Worlds Interpretation of Quantum Theory", Tel-Aviv University preprint, TAUP 2058-93.

Vaidman L. (1994), "Teleportation of Quantum States", Phys. Rev. A, February.

(a)
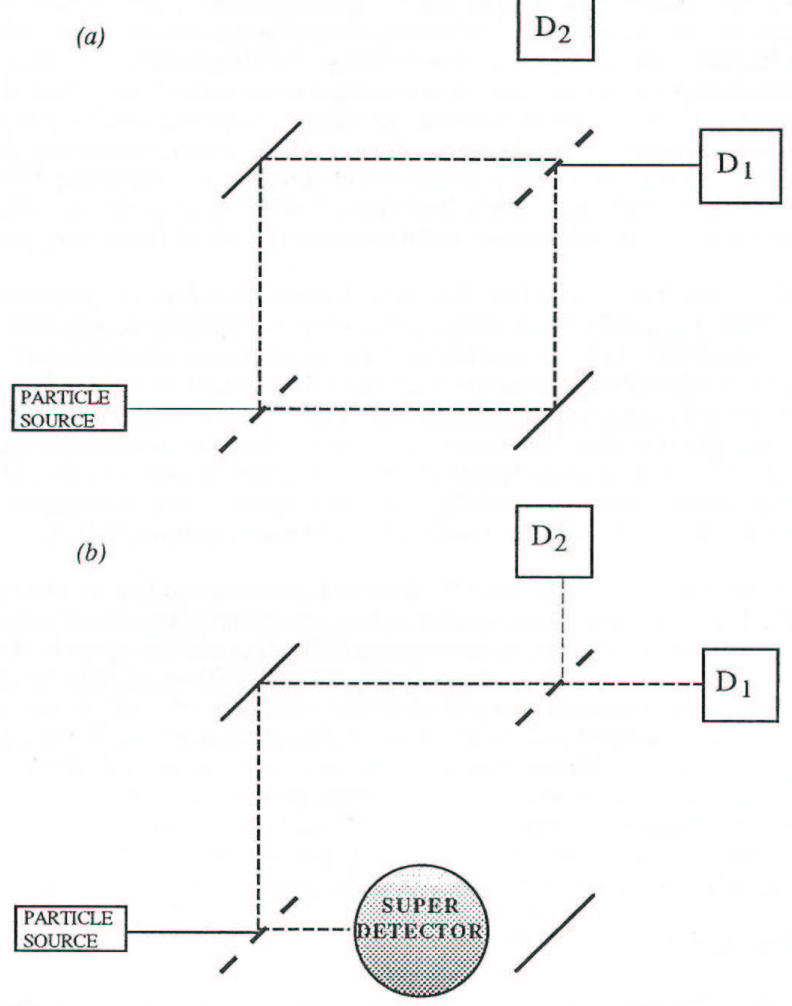

FIG. 1. (a) If there is no any object inside the interferometer, $D_{2}$ never clicks. (b). When $D_{2}$ clicks after sending just one particle we know that the super-detector is inside the interferometer while it did not count any particle. 